\title{
Escala Reduzida de Descritores dos Cinco Grandes Fatores de Personalidade: Prós e Contras
}

\author{
Jean Carlos Natividade \\ Pontifícia Universidade Católica do Rio de Janeiro \\ Rio de Janeiro, RJ, Brasil \\ Claudio Simon Hutz \\ Universidade Federal do Rio Grande do Sul \\ Porto Alegre, RS, Brasil
}

\begin{abstract}
RESUMO
Os objetivos desta pesquisa foram buscar evidências de validade e precisão de uma escala reduzida para aferir características de personalidade sob a perspectiva dos cinco grandes fatores. Dois estudos foram conduzidos sequencialmente. No primeiro, elaborou-se uma escala de 20 itens e verificou-se adequação estrutural. No Estudo 2 , testaram-se relações entre o instrumento elaborado e um teste padronizado que acessa a personalidade no modelo dos cinco grandes fatores. Observaram-se correlações moderadas entre os fatores da medida reduzida e seus correspondentes no teste padronizado. Por fim, testaram-se dois modelos preditivos para a satisfação de vida; um com os fatores da escala reduzida e outro com os do teste padronizado. Ambos os modelos apresentaram os mesmos fatores como explicativos, porém, a medida reduzida explicou apenas metade da variância explicada pelo teste padronizado. Discute-se que mesmo que instrumentos reduzidos apresentem propriedades psicométricas adequadas, eles podem aumentar as chances de erros inferenciais.
\end{abstract}

Palavras-chave: Medidas da personalidade; Traços de personalidade; Construção do teste; Validade do teste.

\section{ABSTRACT}

Short Form Scale of Descriptors of the Five Personality Factors: Pros and Cons

The aims of this research were to seek evidence of validity and precision of a short scale to assess personality characteristics from the perspective of the Big Five factors. Two sequential studies were conducted. In the first, a 20 -item scale was created, and structural adequacy was found. In Study 2, the relations between the short scale and a standardized test to assess personality in the big-five model were tested. Moderate correlations among the short scale factors and their counterparts in the standardized test were noted. Finally, two predictive models for life satisfaction were tested, one with personality factors from the short scale and another with factors from the standardized test. Both models showed the same factors as explanatory. Nevertheless, the short instrument explained only half of the variance explained by the standardized test. We discussed that, even if short scales have suitable psychometric properties, they may increase the odds of inferential errors.

Keywords: Personality measures; Personality traits; Test construction; Test validity.

\section{RESUMEN}

\section{Escala Reducida de Descriptores de los Cinco Grandes Factores de Personalidad: Pros y Contras}

Los objetivos de esta investigación fueron buscar evidencias de validad y precisión de una escala reducida para evaluar características de personalidad desde la perspectiva de los cinco grandes factores. Se realizaron dos estudios secuencialmente. En primero, se elaboró una escala de 20 ítems y se comprobó su adecuación estructural. En el Estudio 2, se verificaron las relaciones entre el instrumento elaborado y un test estandarizado de la personalidad en el modelo de los cinco grandes factores. Se observaron correlaciones moderadas entre los factores de la medida reducida y sus correspondientes en el test estandarizado. Finalmente, se analizaron dos modelos predictivos para la satisfacción de la vida; uno con los factores de la escala reducida y otro con el test estandarizado. Los dos modelos mostraron los mismos factores como explicativos, sin embargo, la medida reducida explicó sólo la mitad de la varianza explicada por el test estandarizado. Se discute que aunque medidas reducidas muestren propiedades psicométricas adecuadas, pueden aumentar las probabilidades de errores inferenciales.

Palabras clave: Medidas de la personalidad; Rasgos de personalidad; Construcción de test; Validación de test. 
O estudo da personalidade tem sido conduzido a partir de enfoques teóricos diversos (Barenbaum \& Winter, 2010). Dentre esses, a perspectiva dos cinco grandes fatores destaca-se pela consensualidade que vem alcançando (John, Nauman, \& Soto, 2010). Essa abordagem compreende a personalidade segundo cinco fatores independentes (Goldberg, 1993). Tais fatores são interpretados como se referindo a contínuos que englobam, resumidamente, os seguintes aspectos: Extroversão - uma tendência a buscar estimulação na interação com outros, a ser ativo e comunicativo; Socialização - uma tendência a demonstrar empatia, altruísmo e comportamentos pró-social; Realização uma tendência ao autocontrole na realização de tarefas que conduzem a um objetivo, a ser disciplinado e organizado; Neuroticismo - uma tendência a demonstrar instabilidade emocional, a experimentar emoções negativas, ansiedade, depressão; Abertura - uma tendência a experimentar coisas novas, a demonstrar curiosidade e complexidade intelectual (John et al., 2010; Nunes, Hutz, \& Nunes, 2010).

Originalmente os cinco grandes fatores denotaram uma descoberta empírica isenta de explicações teóricas a respeito de como e porque essas cinco dimensões representariam adequadamente a personalidade (Goldberg, 1993). Posteriormente, fundamentados na descoberta empírica, Costa e McCrae (1992) desenvolveram um modelo explicativo que ficou conhecido como Modelo dos Cinco Grandes Fatores. A existência ou não de modelos explicativos para os cinco grandes configura uma distinção entre perspectivas concernentes ao tema (Block, 2010; Saucier \& Goldberg, 1996). De um lado está a perspectiva dos cinco grandes como uma síntese descritiva de características pessoais que assume o caráter exploratório dos achados, também chamada de perspectiva léxica (e.g. Saucier \& Goldberg, 2001); de outro lado há uma elaboração teórica explicativa para os cinco grandes fatores em função de disposições biológicas (e.g. Costa \& McCrae, 1992). Dessas visões sobre os cinco grandes fatores decorrem diferenciações que vão desde a nomenclatura dos fatores até a replicabilidade do sistema em outras culturas (Block, 2010). Apesar das diferenças, ambas as perspectivas concordam que os cinco grandes fatores não abarcam todas as peculiaridades da personalidade humana (Saucier \& Goldberg, 1996; McCrae, 2013).

A história da origem dos cinco grandes fatores já foi contada muitas vezes (e.g. Goldberg, 1993; John et al., 2010; Nunes \& Hutz, 2005). Além do destaque para a descoberta empírica corroborada por diversos pesquisadores, ressalta-se na história o pressuposto que guiou os estudos iniciais: que as características pessoais de relevância social e importantes para a vida seriam codificadas em palavras nas línguas faladas pelos povos (uma revisão histórica acerca da abordagem lexical pode ser vista em John, Angleitner, \& Ostendorf, 1988). Sob essa abordagem, realizaram-se buscas em dicionários a fim de selecionar termos, geralmente adjetivos, descritores da personalidade. Posteriormente, análises fatoriais realizadas com as respostas a seleções de termos conduziram o desenvolvimento de instrumentos para avaliar a personalidade na perspectiva dos cinco grandes fatores (John et al., 2010).

Os resultados provenientes de respostas a termos selecionados em dicionários de diferentes culturas (emic studies) não têm sido unânimes em mostrar uma estrutura de cinco fatores (Saucier \& Goldberg, 2001). Por exemplo, a partir do dicionário hebraico considerou-se adequada uma solução de sete fatores como representativos da personalidade (Almagor, Tellegen, \& Waller, 1995); em holandês foram considerados oito fatores (De Raad \& Barelds, 2008); em grego, seis fatores (Saucier, Georgiades, Tsaousis, \& Goldberg, 2005). No Brasil, Pinho e Guzzo (2003) realizaram um levantamento em dicionário de português do Brasil e classificaram 938 adjetivos como descritores da personalidade, mas não se tem notícias que esses adjetivos tenham sido postos à prova empírica.

Por outro lado, quando se parte de traduções de instrumentos do inglês e adapta-se para outras culturas (etic studies), os resultados são mais promissores em apontar para a estrutura penta-fatorial (McCrae et al., 2005). Hutz et al. (1998), por exemplo, inspiraram-se nos marcadores de Goldberg (1992) e Norman (1963), em inglês, e em seus próprios levantamentos com universitários para desenvolver uma lista de adjetivos descritores de personalidade em português do Brasil. Os autores partiram de uma lista de 163 adjetivos, lista essa que foi reduzida a 93 adjetivos após estudos pilotos e, finalmente, reduzida a 64 adjetivos com as análises fatoriais. Os autores consideraram ter encontrado uma adequada estrutura de cinco grandes fatores para a realidade brasileira.

Os instrumentos elaborados para aferir os cinco grandes fatores, pelo menos os mais utilizados, demandam razoável tempo dos respondentes, em função da grande quantidade de itens. Entre os instrumentos mais difundidos, o NEO-PI-R (Costa \& McCrae, 1992) tem 240 itens em formato de afirmativas. Esse instrumento avalia os cinco grandes e 30 facetas relacionadas aos fatores. Os marcadores unipolares para os cinco fatores de Goldberg (1992) contém 100 itens em formato de adjetivos. O BFI (Big Five Inventory) contém 44 itens em formato de afirmativas (John et al., 2010). A Bateria Fatorial de Personalidade, instrumento construído para a 
realidade brasileira, tem 126 itens em formato de afirmativas e afere, além dos cinco grandes fatores, 17 facetas (Nunes et al., 2010). Sobretudo em função do tempo demandado, medidas reduzidas para acessar a personalidade sob a perspectiva dos cinco grandes fatores têm sido desenvolvidas como alternativas para uso em pesquisa (e.g. Gosling, Rentfrow, \& Swann, 2003; Hauck, Machado, Teixeira, \& Bandeira, 2012; Rammstedt \& John, 2007).

Recentemente, Carvalho, Nunes, Primi e Nunes (2012) conduziram uma pesquisa com adolescentes brasileiros para buscar evidências de validade do Ten-Item Personality Inventory (Gosling et al., 2003). Os autores concluíram que o instrumento não apresentava indicadores de validade relativos à estrutura. Hauck, Machado et al. (2012) acharam resultados diferentes para seu instrumento de 25 itens construído com adjetivos do estudo de Hutz et al. (1998). Esses autores consideraram ter encontrado provas satisfatórias para a estrutura de cinco fatores, entre adultos. Posteriormente, os autores testaram a medida em uma amostra de adolescentes e consideraram adequado reter 20 itens para manter a estrutura intacta (Hauck, Texeira, Machado, \& Bandeira, 2012). Essa mesma equipe de pesquisadores, ainda, analisou as propriedades dos itens de seu instrumento para adultos e concluiu que os resultados reforçam os indícios de validade, porém apenas uma estreita faixa do traço latente estaria sendo avaliada (Machado, Hauck, Texeira, \& Bandeira, no prelo).

Instrumentos com poucos itens para avaliar os cinco grandes fatores podem ser muito úteis em contextos de pesquisa em que, mais do que características de personalidade, pretende-se investigar outras tantas variáveis. Medidas menores em termos de tempo de resposta reduzem as chances de fadiga e perda de participantes no procedimento de coleta dos dados. Em delineamentos de pesquisa em que a personalidade não é o alvo principal, instrumentos reduzidos para acessar os cinco grandes fatores, desde que confiáveis, podem fornecer informações importantes que venham a contribuir com a explicação dos achados empíricos. Ao mesmo tempo, esses instrumentos podem apresentar limitações que conduzam a conclusões equivocadas (Credé, Harms, Niehorster, \& Gaye-Valentine, 2012). Levando em conta essa possibilidade e a escassez de investigações que comparam instrumentos reduzidos com testes padronizados para aferir os cinco grandes fatores, desenvolveu-se essa pesquisa com os objetivos de (1) elaborar e verificar adequação estrutural e precisão de uma escala reduzida para aferir os cinco grandes fatores de personalidade; (2) buscar evidências de validade convergente e preditiva do instrumento, comparando-o com um teste padronizado e construído para a realidade brasileira.

\section{ESTUDO 1 \\ ELABORAÇÃO DE ITENS, ESTRUTURA E PRECISÃO}

\section{MÉTODO}

\section{Participantes}

Participaram 1889 adultos residentes das cinco regiões do Brasil, $62,5 \%$ eram mulheres, média de idade de 27,8 anos $(D P=8,86)$. A escolaridade variou de ensino médio completo a ensino de pós-graduação completo: $48,3 \%$ tinham ensino superior completo, $47,9 \%$ tinham superior incompleto e $3,8 \%$ o ensino médio completo. A região do país com mais participantes foi a Sul com $49,5 \%$ dos participantes, a região Nordeste teve $28,4 \%$, a Sudeste teve $15,2 \%$, a Norte teve $2,8 \%$ e a CentroOeste teve 2,6\% dos participantes, os demais $1,5 \%$ declararam estar fora do Brasil.

\section{Instrumentos}

Utilizou-se um questionário on-line disponibilizado em um endereço na internet. Além de questões sociodemográficas, tais como: sexo, idade, escolaridade e local de residência, incluiu-se no questionário a Escala Reduzida de Descritores de Personalidade (Red5). Essa escala foi elaborada neste estudo a fim de avaliar características de personalidade na perspectiva dos cinco grandes fatores: extroversão, socialização, realização, neuroticismo e abertura a experiências. A escala consiste em 20 adjetivos ou pequenas expressões seguidas de uma régua de sete pontos para que os participantes respondam o quanto concordam que cada adjetivo ou expressão os descreve adequadamente.

\section{Procedimentos}

\section{Elaboração dos itens.}

Inspirou-se no levantamento brasileiro de Hutz et al. (1998) e no instrumento de Gosling et al. (2003) para elaboração dos itens; porém, diferentemente desses últimos autores, utilizou-se apenas um adjetivo ou expressão por item. Partindo-se desses dois estudos e de definições dos cinco grandes fatores, elaboraram-se quatro itens para representar cada fator. Os itens que compuseram a versão final do instrumento foram consensualmente escolhidos por dois pesquisadores, ambos psicólogos com experiência em avaliação da personalidade e conhecimento sobre os cinco grandes fatores. Quando havia dúvidas relacionadas à inclusão de algum item enquanto representativo de um fator, 
solicitava-se o auxílio de uma terceira pessoa. Os itens consistiam em adjetivos ou pequenas expressões, por exemplo: amigável, comunicativa(o), responsável, emocionalmente estável, que tem curiosidade. Incluíram-se dois pares de itens opostos para cada fator, a fim de manter a semelhança com o instrumento de Gosling et al (2003). Os adjetivos biformes foram apresentados no feminino e incluíram-se as desinências masculinas entre parênteses, com intuito de evitar viés de gênero (cf. Natividade, Barros, \& Hutz, 2012).

\section{Coleta e análises}

A coleta de dados foi executada inteiramente via internet. Os participantes foram recrutados por meio de e-mails-convites que continham o link para acessar o questionário na internet. Também se disponibilizaram convites com o endereço do questionário em sites de redes sociais. Como critério para participação solicitava-se que os interessados fossem brasileiros com 18 ou mais anos de idade. Ainda, convidaram-se aqueles que completaram o questionário a participar de um segundo momento da pesquisa, a fim de se obterem dados da estabilidade do instrumento. Para tanto, enviaram-se e-mails-convites aos que concordaram em participar dessa segunda etapa, em cerca de seis meses após a primeira resposta, e solicitou-se que respondessem novamente a Escala Reduzida de Descritores de Personalidade (Red5). Para as análises realizou-se uma limpeza na planilha de dados excluindo-se as respostas incompletas e nitidamente falseadas, por exemplo, mesma resposta para todos os itens.

\section{RESULTADOS}

A fim de caracterizar de modo exploratório a estrutura do instrumento elaborado, procedeu-se uma Análise de Componentes Principais, método Varimax de rotação. Constatou-se adequação dos dados à análise, tal que $K M O=0,81$; teste de Bartlett: $\chi^{2}(190, N=1889)=10.811 ; p<0,001$. Observaram-se seis fatores com eigenvalues maiores que 1 , seguem os respectivos valores: 4,$25 ; 2,56 ; 1,84 ; 1,56 ; 1,38$; 1,07. Uma análise paralela de eigenvalues aleatórios revelou que o último eigenvalue observado maior que o simulado foi o da dimensão cinco (sexto eigenvalue simulado $=1,08$; calculou-se com 1889 casos, 20 variá-

TABELA 1

Cargas componenciais dos itens a partir de uma análise de componentes principais com rotação Varimax, Médias, Desvios-Padrões e Coeficientes de Precisão das dimensões

\begin{tabular}{|c|c|c|c|c|c|c|}
\hline & Soc & Ext & Rea & $\mathrm{Neu}$ & Abe & $h^{2}$ \\
\hline simpática(o) & $\mathbf{0 , 8 0}$ & $-0,17$ & 0,04 & $-0,002$ & 0,04 & 0,67 \\
\hline amigável & 0,78 & $-0,07$ & 0,18 & $-0,01$ & 0,12 & 0,66 \\
\hline pouco amigável & $-0,67$ & 0,22 & $-0,07$ & 0,10 & $-0,11$ & 0,52 \\
\hline antipática(o) & $-0,61$ & 0,18 & $-0,04$ & 0,28 & 0,01 & 0,48 \\
\hline tímida(o) & $-0,03$ & $\mathbf{0 , 8 3}$ & $-0,02$ & 0,08 & $-0,10$ & 0,70 \\
\hline calada(o) & $-0,23$ & $\mathbf{0 , 8 1}$ & $-0,02$ & $-0,02$ & $-0,07$ & 0,71 \\
\hline comunicativa(o) & 0,45 & $-0,68$ & 0,08 & 0,10 & 0,11 & 0,69 \\
\hline extrovertida(o) & 0,44 & $-0,67$ & 0,02 & 0,15 & 0,09 & 0,67 \\
\hline responsável & 0,17 & 0,002 & 0,78 & 0,02 & 0,03 & 0,64 \\
\hline esforçada(o) & 0,20 & $-0,01$ & 0,73 & 0,11 & 0,16 & 0,61 \\
\hline indisciplinada(o) & $-0,01$ & $-0,004$ & $-0,70$ & 0,19 & 0,11 & 0,54 \\
\hline desorganizada(o) & 0,07 & 0,11 & $-0,67$ & 0,23 & 0,04 & 0,52 \\
\hline temperamental & $-0,09$ & $-0,06$ & $-0,04$ & 0,75 & $-0,01$ & 0,57 \\
\hline $\operatorname{ansiosa}(0)$ & 0,11 & 0,09 & $-0,002$ & 0,74 & $-0,04$ & 0,57 \\
\hline emocionalmente estável & 0,14 & $-0,01$ & 0,26 & $-0,59$ & 0,04 & 0,44 \\
\hline tranquila(o) & 0,30 & 0,36 & 0,02 & $-0,58$ & 0,05 & 0,56 \\
\hline aberta(o) a novas experiências & 0,19 & $-0,04$ & 0,03 & $-0,03$ & 0,77 & 0,63 \\
\hline que não gosta de mudanças & $-0,04$ & 0,17 & $-0,04$ & 0,19 & $-0,66$ & 0,50 \\
\hline que tem curiosidade & 0,15 & 0,07 & 0,15 & 0,16 & 0,63 & 0,47 \\
\hline convencional & 0,12 & 0,17 & 0,25 & 0,04 & $-0,58$ & 0,45 \\
\hline Eigenvalue & 4,25 & 2,56 & 1,84 & 1,56 & 1,38 & \\
\hline \% Variância explicada & 13,9 & 12,8 & 11,5 & 10,4 & 9,33 & \\
\hline Média & 5,88 & 4,65 & 5,09 & 4,08 & 4,92 & \\
\hline Desvio-Padrão & 1,04 & 1,48 & 1,16 & 1,26 & 1,09 & \\
\hline Coeficiente Alfa & 0,77 & 0,84 & 0,70 & 0,67 & 0,59 & \\
\hline Teste-reteste $^{\dagger}$ & 0,74 & 0,78 & 0,79 & 0,81 & 0,69 & \\
\hline
\end{tabular}

Nota: Cargas maiores que 0,50 estão em negrito. $N=1889$. Soc: Socialização; Ext: Extroversão; Rea: Realização; Neu: Neuroticismo; Abe: Abertura a experiências.

$\dagger$ Tempo médio teste-reteste: seis meses, $N=100$. 
veis, 1000 amostras bootstrap). Considerando o critério de Horn (1965) para retenção de fatores e a literatura a respeito dos cinco fatores de personalidade, extraíram-se cinco componentes que explicaram $57,9 \%$ da variância dos dados. As cargas dos itens nas dimensões podem ser vistas na Tabela 1. Os itens carregaram mais fortemente nas respectivas dimensões para as quais foram elaborados, sugerindo adequação do instrumento. Em seguida, calcularam-se médias e desvios-padrões para cada dimensão invertendo-se os itens com carga negativa. Também na Tabela 1 são apresentados os coeficientes de consistência interna e correlação testereteste. Os indicadores de precisão tomados em conjunto sugerem razoável precisão da escala.

\section{ESTUDO 2 RELAÇÕES COM OUTRAS VARIÁVEIS}

\section{MÉTODO}

\section{Participantes}

Foram participantes 512 adultos, dentre os quais 58\% eram mulheres, média de idade de 28,6 anos $(D P=7,90)$. Quanto à escolaridade, $56,8 \%$ dos participantes tinham ensino superior completo, $38,1 \%$ tinham superior incompleto e $5,1 \%$ ensino médio completo. A maioria dos participantes deste estudo era da região Sul do país, $87,5 \%$, a segunda região com maior número de participantes foi a região Sudeste com $8,6 \%$, os demais $3,9 \%$ dos participantes estavam entre as três outras regiões do país ou fora do país.

\section{Instrumentos}

Utilizou-se um questionário on-line disponibilizado em um endereço na internet, semelhante ao do Estudo 1. Além das questões sociodemográficas (sexo, idade, escolaridade e local de residência) e da Escala Reduzida de Descritores de Personalidade - Red5 (elaborada no Estudo 1), o questionário continha a Bateria Fatorial de Personalidade - BFP (Nunes et al., 2010) e a Escala de Satisfação de Vida - SV (Giacomoni \& Hutz, 1997). A BFP é um teste elaborado, padronizado e normatizado para o Brasil para acessar a personalidade no modelo dos cinco grandes fatores. A bateria é formada por 126 itens em formato de afirmativas para que os participantes respondam em uma escala de sete pontos o quanto cada uma os descreve adequadamente. $\mathrm{O}$ instrumento ainda permite aferir 17 facetas relacionadas aos fatores. A Escala de Satisfação de Vida, adaptada para o Brasil a partir da versão de Diener, Emmons, Larsen e Griffin (1985), afere aspectos cognitivos globais do bem estar subjetivo. Ela é composta por cinco itens em formato de afirmativas seguidas de uma escala de sete pontos para que os participantes respondam o quanto concordam com cada uma delas.

\section{Procedimentos}

Os procedimentos de coleta e análises dos dados foram idênticos aos do Estudo 1. Recrutaram-se participantes enviando-se e-mails-convites e disponibilizaram-se convites em redes sociais. Para as análises, realizou-se a limpeza dos dados retirando-se os casos omissos às respostas da Red5 e BFP e os dados possivelmente falseados.

\section{RESULTADOS}

Inicialmente, testou-se a adequação dos dados à estrutura do instrumento por meio de uma Análise Fatorial Confirmatória, utilizando-se o software AMOS 16.0 para especificação do modelo e cálculo dos índices de ajuste. O modelo foi configurado utilizando-se a técnica de agrupamento de itens (Little, Cunningham, Shahar, \& Widaman, 2002); assim, cada fator foi composto por duas observáveis. Cada observável correspondia à soma dos escores de dois itens representativos do fator, sendo um deles invertido. A configuração do modelo pode ser descrita como cinco variáveis latentes correlacionadas e cada uma delas explicando duas observáveis resultantes da agregação de pares de itens. Entre as vantagens do uso dessa técnica destacam-se, sobretudo, a redução dos efeitos da distribuição anômala e compartilhamento da variância de erros de itens na estimação dos parâmetros do modelo (Little et al., 2002).

Para o cálculo dos parâmetros partiu-se da matriz de covariância e optou-se pelo algoritmo Maximum Likelihood. Levaram-se em conta os seguintes índices para verificação do ajuste do modelo, tal como tem sido sugerido pela literatura (Byrne, 2009): razão entre qui-quadrado e graus de liberdade; Goodnessof-Fit Index (GFI); Adjusted Goodness-of-Fit Index (AGFI); Comparative Fit Index (CFI); Normed Fit Index (NFI); Root Mean Square Error of Aproximation (RMSEA). O modelo especificado obteve os seguintes índices de ajuste: $\chi^{2}(25, N=512)=164,8 ; p<0,001 \mathrm{e}$ $\chi^{2} / d f=6,59 ; \quad \mathrm{GFI}=0,98 ; \quad \mathrm{AGFI}=0,96 ; \quad \mathrm{NFI}=0,97$; $\mathrm{TLI}=0,95 ; \mathrm{CFI}=0,97 ; \mathrm{RMSEA}=0,054 ;$ Intervalo de Confiança de 90\% para RMSEA=0,047-0,062. Adicionalmente, testou-se o ajuste do modelo também com um método de estimação robusto (Satorra \& Bentler, 1994). Para isso, utilizou-se o software R (R Development Core Team, 2012) e o pacote estatístico Lavaan (Rossel, 2012). Os resultados para o método robusto foram bastante semelhantes aos encontrados 
com o método Maximum Likelihood, por exemplo, obteve-se $\chi^{2}(25, N=512)=145,2 ; p<0,001 ; \chi^{2} / d f=5,81$; $\mathrm{TLI}=0,95 ; \mathrm{CFI}=0,97$ e RMSEA $=0,050$. Pode-se considerar que esses índices, tomados em conjunto, apontam para a adequação da estrutura do instrumento (c.f. Byrne, 2009).

O primeiro passo dado no sentido de comparar o instrumento aqui construído (Red5) com um teste padrão para avaliar os cinco grandes fatores (BFP) foi calcular as médias e os desvios-padrões dos fatores de personalidade avaliados por ambos. A Tabela 2 mostra os cálculos descritivos para os instrumentos. É possível notar que, apesar dos instrumentos compartilharem a mesma grade de respostas, as médias e os desvios-padrões entre eles diferem consideravelmente. Os desvios do Red5 são mais elevados para todos os fatores, o que indica maior variabilidade nas respostas a esse instrumento. Outra diferença que se destaca diz respeito a menor precisão do Red5 quando comparado à BFP, tal como indicam os coeficientes alfa menores daquele instrumento.

Em seguida, testaram-se diferenças sexuais nos fatores de personalidade utilizando-se ambos os instrumentos. Excetuando-se o fator Realização, todos os demais apresentaram diferenças, ou ausência de diferença, no mesmo sentido nos dois instrumentos. A Tabela 2 mostra os tamanhos de efeito das diferenças. Observa-se que para o fator Extroversão o tamanho das diferenças nos dois instrumentos mostrou-se virtualmente idêntico.

Outros passos tomados a fim de comparar os dois instrumentos foram realizados com testes de correlações. Inicialmente, calculou-se o coeficiente de correlação canônica (Knapp, 1978) entre os dois conjuntos de variáveis, os cinco fatores de cada instrumento, e encontrou-se um coeficiente de 0,80. Em seguida, calcularam-se os coeficientes de correlação múltipla entre as facetas de cada fator acessado pela BFP e o fator correspondente avaliado pela Red5, tais que foram os resultados: Socialização, $R=0,59$; Extroversão, $R=0,81$; Realização, $R=0,55$; Neuroticismo, $R=0,66$; Abertura, $R=0,56$. Por fim, elaborou-se uma matriz de correlações entre os fatores do Red5 e os fatores e subfatores da BFP. A Tabela 3 mostra os coeficientes de Pearson. Observa-se que os coeficientes foram mais elevados entre os fatores e facetas da BFP e seus respectivos correspondentes da Red5.

Os últimos passos dados a fim comparar o instrumento elaborado neste trabalho (Red5) com um teste padrão para acessar a personalidade no modelo dos cinco grandes fatores (BFP) foram no sentido de testar a capacidade preditiva dos dois instrumentos para o construto Satisfação de Vida (SV). Para tanto, realizaram-se análises de regressão linear múltipla incluindo-se os fatores de cada instrumento como preditores e a Satisfação de Vida como variável desfecho. Testaram-se dois modelos explicativos, um para cada instrumento. Os resultados podem ser vistos na Tabela 4. Observa-se que as mesmas variáveis dos dois modelos explicaram significativamente a SV e no mesmo sentido, contudo a variância explicada pela BFP foi quase o dobro da explicada pela Red5. Além disso, realizou-se uma análise de regressão múltipla hierárquica em que se incluíram no primeiro passo os fatores da BFP e no segundo os fatores da Red5. Os resultados dessa última análise podem ser vistos na Tabela 5. Nota-se que houve um acréscimo significativo, ainda que pequeno (de $2 \%$ ), de variância explicada com a inclusão da Red5.

TABELA 2

Dados psicométricos dos cinco grandes fatores para a Bateria Fatorial de Personalidade e Escala de Descritores Reduzidos de Personalidade, Coeficientes Alfa e diferenças sexuais

\begin{tabular}{|c|c|c|c|c|c|c|c|c|c|c|}
\hline & \multicolumn{2}{|c|}{ Socialização } & \multicolumn{2}{|c|}{ Extroversão } & \multicolumn{2}{|c|}{ Realização } & \multicolumn{2}{|c|}{ Neuroticismo } & \multicolumn{2}{|c|}{ Abertura } \\
\hline & $B F P$ & Red5 & $B F P$ & Red5 & $B F P$ & Red5 & $B F P$ & Red5 & $B F P$ & Red5 \\
\hline Número itens & 28 & 4 & 25 & 4 & 21 & 4 & 29 & 4 & 23 & 4 \\
\hline Coeficiente Alfa & 0,84 & 0,80 & 0,88 & 0,81 & 0,82 & 0,67 & 0,90 & 0,66 & 0,80 & 0,52 \\
\hline Mínimo & 2,58 & 2,33 & 1,57 & 1 & 2,74 & 1,67 & 1,28 & 1 & 2,38 & 1,33 \\
\hline Máximo & 6,67 & 7 & 6,29 & 7 & 6,82 & 7 & 6,15 & 7 & 7 & 7 \\
\hline $\begin{array}{l}\text { Média Todos } \\
\text { (Desvio-Padrão) }\end{array}$ & $\begin{array}{c}5,16 \\
(0,67)\end{array}$ & $\begin{array}{c}5,53 \\
(0,96)\end{array}$ & $\begin{array}{c}4,32 \\
(0,85)\end{array}$ & $\begin{array}{c}4,54 \\
(1,37)\end{array}$ & $\begin{array}{c}4,91 \\
(0,75)\end{array}$ & $\begin{array}{c}5,59 \\
(0,99)\end{array}$ & $\begin{array}{c}3,28 \\
(0,89)\end{array}$ & $\begin{array}{c}4,25 \\
(1,19)\end{array}$ & $\begin{array}{c}4,68 \\
(0,76)\end{array}$ & $\begin{array}{c}4,44 \\
(1,13)\end{array}$ \\
\hline$d$ Diferença BFP - Red5 & \multicolumn{2}{|c|}{$0,45^{* * *}$} & \multicolumn{2}{|c|}{$0,20 * * *$} & \multicolumn{2}{|c|}{$0,78 * * *$} & \multicolumn{2}{|c|}{$0,94 * * *$} & \multicolumn{2}{|c|}{$0,25^{* * *}$} \\
\hline $\begin{array}{l}\text { Média Homens } \\
\text { (Desvio-Padrão) }\end{array}$ & $\begin{array}{c}5,02 \\
(0,67)\end{array}$ & $\begin{array}{c}5,39 \\
(1,03)\end{array}$ & $\begin{array}{c}4,20 \\
(0,89)\end{array}$ & $\begin{array}{c}4,31 \\
(1,36)\end{array}$ & $\begin{array}{c}4,91 \\
(0,73)\end{array}$ & $\begin{array}{c}5,42 \\
(1,04)\end{array}$ & $\begin{array}{c}3,19 \\
(0,86)\end{array}$ & $\begin{array}{c}3,93 \\
(1,16)\end{array}$ & $\begin{array}{c}4,71 \\
(0,75)\end{array}$ & $\begin{array}{c}4,44 \\
(1,13)\end{array}$ \\
\hline $\begin{array}{l}\text { Média Mulheres } \\
\text { (Desvio-Padrão) }\end{array}$ & $\begin{array}{c}5,27 \\
(0,66)\end{array}$ & $\begin{array}{c}5,64 \\
(0,89)\end{array}$ & $\begin{array}{c}4,41 \\
(0,81)\end{array}$ & $\begin{array}{c}4,70 \\
(1,35)\end{array}$ & $\begin{array}{c}4,90 \\
(0,77)\end{array}$ & $\begin{array}{c}5,71 \\
(0,93)\end{array}$ & $\begin{array}{c}3,34 \\
(0,91)\end{array}$ & $\begin{array}{c}4,48 \\
(1,16)\end{array}$ & $\begin{array}{c}4,66 \\
(0,76)\end{array}$ & $\begin{array}{c}4,44 \\
(1,13)\end{array}$ \\
\hline$d$ Diferença Sexual $^{\dagger}$ & $-0,37 * * *$ & $-0,26 * *$ & $-0,25^{* *}$ & $-0,29 * *$ & 0,01 & $-0,30 * *$ & $-0,18^{*}$ & $-0,48 * * *$ & 0,06 & $-0,001$ \\
\hline
\end{tabular}

Nota: Para as diferenças de médias entre BFP e Red5 utilizaram-se testes $t$ para amostras repetidas; para as diferenças sexuais testes $t$ para amostras independentes. BFP: Bateria Fatorial de Personalidade; Red5: Escala Reduzida de Descritores de Personalidade. $N=512$.

$* p=0,05 ; * * p<0,01 ; * * * p<0,001, \uparrow$ sinais negativos indicam médias maiores para as mulheres, tamanho de efeito (Cohen, 1962). 
TABELA 3

Correlações entre fatores da Escala Reduzida de Descritores de Personalidade (Red5) e os fatores e facetas da Bateria Fatorial de Personalidade (BFP)

\begin{tabular}{|c|c|c|c|c|c|}
\hline & $\begin{array}{l}\text { Socialização } \\
\text { (Red5) }\end{array}$ & $\begin{array}{l}\text { Extroversão } \\
\text { (Red5) }\end{array}$ & $\begin{array}{l}\text { Realização } \\
\text { (Red5) }\end{array}$ & $\begin{array}{c}\text { Neuroticismo } \\
\text { (Red5) }\end{array}$ & $\begin{array}{c}\text { Abertura } \\
\text { (Red5) }\end{array}$ \\
\hline Socialização (BFP) & $0,53 * *$ & $0,14 * *$ & $0,34 * *$ & $-0,24 * *$ & 0,03 \\
\hline Amabilidade & $0,53 * *$ & $0,12 * *$ & $0,23 * *$ & $-0,05$ & $0,10 *$ \\
\hline Pró-sociabilidade & $0,25 * *$ & 0,01 & $0,43 * *$ & $-0,10^{*}$ & $-0,23 * *$ \\
\hline Confiança nas pessoas & $0,39 * *$ & $0,19 * *$ & $0,10^{*}$ & $-0,34 * *$ & $0,19 * *$ \\
\hline Extroversão (BFP) & $0,42 * *$ & $0,70 * *$ & 0,04 & 0,05 & $0,32 * *$ \\
\hline Comunicação & $0,38 * *$ & $0,81 * *$ & 0,08 & 0,01 & $0,28 * *$ \\
\hline Altivez & 0,05 & $0,34 * *$ & $-0,16^{* *}$ & $0,28 * *$ & $0,10^{*}$ \\
\hline Dinamismo & $0,37 * *$ & $0,45 * *$ & $0,23 * *$ & $-0,15^{* *}$ & $0,28 * *$ \\
\hline Interações sociais & $0,48 * *$ & $0,55^{* *}$ & $-0,03$ & 0,02 & $0,32 * *$ \\
\hline Realização (BFP) & $0,16^{* *}$ & $-0,14^{* *}$ & $0,51 * *$ & $-0,13^{* *}$ & $-0,06$ \\
\hline Competência & $0,24 * *$ & $0,14 * *$ & $0,48 * *$ & $-0,09 *$ & $0,19 * *$ \\
\hline Prudência & $0,11^{*}$ & $-0,25^{* *}$ & $0,25 * *$ & $-0,28 * *$ & $-0,15^{* *}$ \\
\hline Comprometimento & 0,03 & $-0,14 * *$ & $0,46^{* *}$ & $0,09 *$ & $-0,11^{*}$ \\
\hline Neuroticismo (BFP) & $-0,29 * *$ & $-0,25^{* *}$ & $-0,39 * *$ & $0,50 * *$ & $-0,26 * *$ \\
\hline Vulnerabilidade & $-0,19 * *$ & $-0,36^{* *}$ & $-0,18^{* *}$ & $0,32 * *$ & $-0,31 * *$ \\
\hline Instabilidade emocional & $-0,27 * *$ & $-0,07$ & $-0,23 * *$ & $0,65^{* *}$ & $-0,15^{* *}$ \\
\hline Passividade & $-0,17 * *$ & $-0,10^{*}$ & $-0,56^{* *}$ & $0,19 * *$ & $-0,10 *$ \\
\hline Depressão & $-0,29 * *$ & $-0,30 * *$ & $-0,26^{* *}$ & $0,34 * *$ & $-0,26^{* *}$ \\
\hline Abertura (BFP) & $0,12 * *$ & $0,11^{*}$ & $-0,15^{* *}$ & $-0,07$ & $0,51 * *$ \\
\hline Ideias & 0,08 & $0,12 * *$ & $-0,07$ & $-0,09 *$ & $0,33 * *$ \\
\hline Liberalismo & 0,02 & 0,03 & $-0,10^{*}$ & $-0,06$ & $0,26^{* *}$ \\
\hline Busca por novidades & $0,16^{* *}$ & $0,10^{*}$ & $-0,17 * *$ & $-0,003$ & $0,53 * *$ \\
\hline
\end{tabular}

Nota: Os coeficientes de correlações entre os fatores da Bateria Fatorial de Personalidade (BFP) e seus correspondentes na Escala Reduzida de Descritores de Personalidade (Red5) estão em negrito. $N=512$.

$* p<0,05 ; * * p<0,01$.

\section{TABELA 4}

Modelos explicativos da satisfação de vida a partir dos cinco fatores de personalidade acessados por dois instrumentos diferentes

\begin{tabular}{|c|c|c|c|c|c|}
\hline & $\beta$ & $t$ & $p$ & $R^{2}$ & $F(5,506)$ \\
\hline & Modelo 1 - Red5 & & & 0,15 & 18,$2 ; p<0,001$ \\
\hline Constante & & 6,39 & $<0,001$ & & \\
\hline Socialização (Red5) & 0,03 & 0,70 & 0,487 & & \\
\hline Extroversão (Red5) & 0,15 & 3,29 & 0,001 & & \\
\hline Realização (Red5) & 0,26 & 6,16 & $<0,001$ & & \\
\hline Neuroticismo (Red5) & $-0,22$ & $-5,15$ & $<0,001$ & & \\
\hline Abertura (Red5) & $-0,02$ & $-0,46$ & 0,643 & & \\
\hline \multicolumn{4}{|c|}{ Modelo 2 - BFP } & 0,28 & 38,$1 ; p<0,001$ \\
\hline Constante & & 5,86 & $<0,001$ & & \\
\hline Socialização (BFP) & 0,03 & 0,82 & 0,414 & & \\
\hline Extroversão (BFP) & 0,15 & 3,60 & $<0,001$ & & \\
\hline Realização (BFP) & 0,16 & 4,05 & $<0,001$ & & \\
\hline Neuroticismo (BFP) & $-0,39$ & $-9,18$ & $<0,001$ & & \\
\hline Abertura (BFP) & 0,01 & 0,15 & 0,881 & & \\
\hline
\end{tabular}

Nota: Modelo 1 - Red5: variáveis explicativas decorrentes da Escala Reduzida de Descritores de Personalidade (Red5). Modelo 2 - BFP: variáveis explicativas decorrentes da Bateria Fatorial de Personalidade (BFP). Ambos os modelos foram obtidos por meio de uma regressão linear, método Enter. $N=507$. 
TABELA 5

Modelo de regressão hierárquica explicativo da satisfação de vida a partir BFP e Red5

\begin{tabular}{|c|c|c|c|c|c|c|c|}
\hline & \multicolumn{3}{|c|}{ Bloco 1} & \multicolumn{3}{|c|}{ Bloco 2} \\
\hline & & $\beta$ & $t$ & $p$ & $\beta$ & $t$ & $p$ \\
\hline Constante & & & 5,86 & $<0,001$ & & 5,90 & $<0,001$ \\
\hline Socialização (BFP) & & 0,03 & 0,82 & 0,41 & 0,04 & 0,93 & 0,36 \\
\hline Extroversão (BFP) & & 0,15 & 3,60 & $<0,001$ & 0,25 & 4,20 & $<0,001$ \\
\hline Realização (BFP) & & 0,16 & 4,05 & $<0,001$ & 0,08 & 1,67 & 0,10 \\
\hline Neuroticismo (BFP) & & $-0,39$ & $-9,18$ & $<0,001$ & $-0,40$ & $-7,53$ & $<0,001$ \\
\hline Abertura (BFP) & & 0,01 & 0,15 & 0,88 & 0,09 & 1,87 & 0,06 \\
\hline Socialização (Red5) & & & & & $-0,05$ & $-0,97$ & 0,33 \\
\hline Extroversão (Red5) & & & & & $-0,08$ & $-1,32$ & 0,19 \\
\hline Realização (Red5) & & & & & 0,08 & 1,64 & 0,10 \\
\hline Neuroticismo (Red5) & & & & & $-0,04$ & $-0,81$ & 0,42 \\
\hline \multirow[t]{5}{*}{ Abertura (Red5) } & & & & & $-0,16$ & $-3,36$ & 0,001 \\
\hline & $R^{2}$ & & 0,28 & & & 0,30 & \\
\hline & $F$ & & $38,1 * * *$ & & & $21,3 * * *$ & \\
\hline & $\Delta R^{2}$ & & & & & 0,02 & \\
\hline & $\Delta F$ & & & & & $3,49 * *$ & \\
\hline
\end{tabular}

Nota: BFP: variáveis explicativas decorrentes da Bateria Fatorial de Personalidade (BFP). Red5: variáveis explicativas decorrentes da Escala Reduzida de Descritores de Personalidade (Red5). Modelo obtido por meio de uma regressão linear hierárquica, método Enter. $N=507$.

$* * p<0,01 ; * * * p<0,001$.

\section{DISCUSSÃO}

Elaborou-se um instrumento reduzido para avaliar os cinco grandes fatores de personalidade e buscaram-se evidências de sua validade. Além de evidências relacionadas ao conteúdo e à estrutura do instrumento, buscaram-se evidências baseadas nas relações com outras variáveis, tal como preconizam os Standards para construção de instrumentos (AERA, APA, \& NCME, 1999). De maneira inédita no Brasil em pesquisas com instrumentos reduzidos para aferir personalidade, realizaram-se procedimentos de comparação com um teste padronizado e construído para a realidade brasileira para acessar os cinco grandes fatores de personalidade. Os resultados apontaram para um instrumento reduzido com satisfatórias evidências de validade. Contudo, as comparações com o teste de personalidade padronizado permitiram constatar limitações importantes para o instrumento reduzido aqui construído, limitações essas que talvez se apliquem a outros instrumentos reduzidos de mesmo propósito (ver também Credé et al., 2012).

A escala construída (Red5) tem 20 itens que se distribuíram de acordo com o previsto na construção do instrumento entre cinco fatores, quatro itens por fator. Essa estrutura foi observada em análise exploratória no Estudo 1 e confirmada no Estudo 2. Os resultados mostraram-se em acordo com a estrutura dos cinco grandes fatores de personalidade e os índices obtidos foram bastante semelhantes a de outros estudos com instrumentos reduzidos (e.g. Hauck, Machado et al., 2012; Hauck, Texeira et al., 2012).

Os índices de precisão, representados pelos coeficientes alfas, sugerem razoável fidedignidade ao instrumento para a maioria dos fatores acessados (cf. Nunnally, 1978). Porém, foram observados índices considerados problemáticos para precisão nos fatores Neuroticismo e Abertura (abaixo de 0,70). Apesar disso, esses índices problemáticos foram semelhantes aos de outros estudos com instrumentos reduzidos (e.g. Gosling et al., 2003; Hauck, Machado et al., 2012; Hauck, Texeira et al., 2012; Rammstedt \& John, 2007). Considera-se importante destacar que algumas limitações da escala podem ter influenciado os índices de consistência interna, seja deflacionando-os em função do baixo número de itens por fator, ou mesmo inflacionando-os em função da presença de itens muito semelhantes aos seus opositores num mesmo fator. Em função dessas limitações, os coeficientes de correlação teste-reteste mostraram-se uma alternativa para caracterizar os níveis de precisão da escala. Uma relativa consistência temporal é um pressuposto dos traços de personalidade e observou-se neste estudo que o instrumento foi capaz de captar essa consistência.

Por outro lado, ao se compararem os coeficientes alfa da Red5 com os da BFP, Estudo 2, observam-se 
resultados consideravelmente inferiores para aquele instrumento nas dimensões Neuroticismo, Realização e Abertura. A esse respeito, além das limitações destacadas acima, é preciso ponderar que o uso de adjetivos descontextualizados em instrumentos como a Red5 permite uma amplitude maior de interpretações de significados para os itens, consequentemente maior probabilidade de erro da medida. As diferenças de precisão entre os instrumentos, bem como as diferenças na complexidade de aplicação e nos resultados que ambos produzem, devem ser levadas em conta na escolha de instrumentos para pesquisas futuras.

As diferenças sexuais encontradas para os fatores de personalidade aferidos pelos dois instrumentos foram no mesmo sentido para todas as dimensões, exceto Realização (Tabela 2). Esses resultados ratificam a validade do instrumento reduzido, tal que mostra resultados semelhantes aos da BFP e em acordo com a literatura. As mulheres apresentaram médias superiores aos homens em Extroversão, Neuroticismo e Socialização, sendo que para esses dois últimos fatores os resultados são semelhantes a estudos em diversas culturas (e.g. Costa, Terracciano, \& McCrae, 2001; Schmitt, Realo, Voracek, \& Allik, 2008; Vianelloa, Schnabel, Sriram, \& Nosek, 2013) e em pesquisas anteriores com a BFP (Nunes et al., 2010). Para o fator Realização verificou-se discrepância entre as duas medidas, sendo que as mulheres apresentaram médias superiores apenas na medida reduzida e não houve diferença quando o fator foi acessado com a BFP. Para o fator Abertura, nenhum dos instrumentos destacou diferenças sexuais. Embora os resultados tenham se mostrado no mesmo sentido para quase todos os fatores, pode-se verificar diferenças acentuadas nos tamanhos de efeito. Considerando-se a BFP o instrumento padrãoouro nesta pesquisa, essas discrepâncias de tamanho de efeito denotam a fragilidade da Red5 e salientam as chances de ocorrerem erros de inferência utilizando-se medidas reduzidas, como também ressaltaram Credé et al. (2012).

A correlação entre BFP e Red5, avaliada pelo coeficiente de correlação canônica, permite concluir que há um compartilhamento de $64 \%$ de variância das duas medidas. Considerando-se a abrangência da BFP e a concisão da Red5, esse resultado pode ser encarado como favorável à Red5. Entretanto, quando se observam as análises de correlações múltiplas entre as facetas da BFP e os fatores relacionados a elas da Red5, os dados já não se mostram tão animadores. $\mathrm{O}$ melhor resultado encontrado foi para a Extroversão, que compartilhou $65,6 \%$ da variância com as facetas de Extroversão da BFP; e o pior foi o da Realização, $30,2 \%$ de variância compartilhada com as facetas de Realização da BFP. Os resultados dos demais fatores ficaram em torno de $31 \%$ a $43 \%$ de variância compartilhada. A matriz de correlações entre as facetas e fatores da BFP e os fatores da Red5 (Tabela 3) acentua as lacunas desse instrumento em cobrir o construto de modo amplo tal como aquele. Apesar disso, verificouse que correlações mais fortes ocorreram entre fatores e subfatores coincidentes da BFP e Red5, o que evidencia a qualidade estrutural da Red5. O que se pode concluir desses resultados é que os fatores da Red5 estão aferindo de maneira desproporcional os subfatores de cada grande fator.

Os resultados da predição da Satisfação de Vida tanto com a Red5, quanto com a BFP, mostraram as mesmas variáveis como explicativas da variância do construto, bem como no mesmo sentido para ambos os instrumentos (Tabela 4). Realização e Extroversão explicaram positivamente a Satisfação de Vida, Neuroticismo explicou negativamente. Esses resultados estão em consonância com outros estudos que investigaram essas relações (e.g. Garcia \& Erlandsson, 2011; Gomez, Krings, Bangerter, \& Grob, 2009; Grant, Langan-Fox, \& Anglim, 2009; Hayes \& Joseph, 2003; Haslam, Whelan, \& Bastian, 2009; Nunes, Hutz, \& Giacomoni, 2009; Woyciekoski, Natividade, \& Hutz, no prelo). Observa-se que a explicação decorrente da BFP é quase o dobro do que é explicado pela Red5. Contudo, a Red5 acrescenta uma parcela significativa de variância, além do que explica a BFP (Tabela 5). Se por um lado o instrumento reduzido apresenta resultados semelhantes aos da BFP e até mesmo pode incrementar a variância explicada sobre outro construto, que fortalecem as evidências em prol de sua validade; por outro lado, o Red5 reduz a capacidade explicativa quase pela metade.

Considera-se que ao mesmo tempo em que se pode advogar em favor do instrumento reduzido aqui elaborado, tendo em vista suas propriedades psicométricas, as comparações com um teste padronizado trazem à tona suas limitações. Essas limitações podem conduzir a conclusões equivocadas sobre resultados de pesquisas que se utilizem do instrumento reduzido, tais como ocorrência de erros inferenciais. Levando-se em conta os resultados deste estudo e aqueles encontrados por Credé et al. (2012) sugere-se cautela no uso dessa e de outras medidas reduzidas para aferir os cinco grandes fatores. Por fim, incentivam-se novas investigações comparativas utilizando-se testes padronizados no Brasil. 


\section{REFERÊNCIAS}

American Educational Research Association, American Psychological Association \& National Council on Measurement in Education (1999). Standards for educational and psychological testing. Washington, DC: American Educational Research Association Publications.

Almagor, M., Tellegen, A., \& Waller, N. G. (1995). The Big Seven model: A cross-cultural replication and further exploration of the basic dimensions of natural language trait descriptors. Journal of Personality and Social Psychology, 69(2), 300-307. http://dx.doi.org/10.1037/0022-3514.69.2.300

Barenbaum, N. B. \& Winter, D. G. (2010). History of modern personality theory and research. In O. P. John, R. W. Robins, \& L. A. Pervin (Eds.), Handbook of personality: Theory and research (3rd ed., pp. 114-158). New York: Guilford Press.

Block, J. (2010). The five-factor framing of personality and beyond: Some ruminations. Psychological Inquiry, 21(1), 2-25. http://dx.doi.org/10.1080/10478401003596626

Byrne, B. M. (2009). Structural equation modeling with AMOS: Basic concepts, applications, and programming (2nd ed.). New York: Routledge.

Carvalho, L. F., Nunes, M. F. O., Primi, R., \& Nunes, C. H. S. S. (2012). Evidências desfavoráveis para avaliação da personalidade com um instrumento de 10 itens. Paidéia (Ribeirão Preto), 22, 63-71. http://dx.doi.org/10.1590/S0103863 X2012000100008

Cohen, J. (1962). The statistical power of abnormal-social psychological research: A review. Journal of Abnormal and Social Psychology, 65(3), 145-153. http://dx.doi.org/10.1037/h0045186

Costa, P. T. \& McCrae, R. R. (1992). Revised NEO Personality Inventory (NEO-PI-R) and NEO Five-Factor Inventory (NEO-FFI) professional manual. Odessa: Psychological Assessment Resources.

Costa, P. T., Terracciano, A., \& McCrae, R. R. (2001). Gender differences in personality traits across cultures: Robust and surprising findings. Journal of Personality and Social Psychology, 81(2), 322-331. http://dx.doi.org/10.1037/00223514.81.2.322

Credé, M., Harms, P., Niehorster, S., \& Gaye-Valentine, A. (2012). An evaluation of the consequences of using short measures of the Big Five personality traits. Journal of Personality and Social Psychology, 102, 874-888. http://dx.doi. org/10.1037/a0027403

De Raad, B. \& Barelds, D. P. H. (2008). A new taxonomy of Dutch personality traits based on a comprehensive and unrestricted list of descriptors. Journal of Personality and Social Psychology, 94, 347-364. http://dx.doi. org/10.1037/0022-3514.94.2.347

Diener, E., Emmons, R., Larsen, R., \& Griffin, S. (1985). The satisfaction with life scale. Journal of Personality Assessment, 49(1), 91-95. http://dx.doi.org/10.1207/s15327752jpa4901_13

Garcia, D. \& Erlandsson, A. (2011). The relationship between personality and subjective well-being: Different association patterns when measuring the affective component in frequency and intensity. Journal of Happiness Studies, 12(6), 1023-1034. http://dx.doi.org/10.1007/s10902-010-9242-6

Giacomoni, C. H. \& Hutz, C. S. (1997). A mensuração do bem-estar subjetivo: escala de afeto positivo e negativo e escala de satisfação de vida [Resumos]. In Sociedade Interamericana de Psicologia (Org.). Anais XXVI Congresso Interamericano de Psicologia (p. 313). São Paulo: SIP.

Goldberg, L. R. (1992). The development of markers for the Big-Five factor structure. Psychological Assessment, 4 , 26-42. http://dx.doi.org/10.1037/1040-3590.4.1.26

Goldberg, L. R. (1993). The structure of phenotypic personality traits. American Psychologist, 48, 26-34. http://dx.doi. org/10.1037/0003-066X.48.1.26

Gomez, V., Krings, F., Bangerter, A., \& Grob, A. (2009). The influence of personality and life events on subjective well-being from a life span perspective. Journal of Research in Personality, 43, 345-354. http://dx.doi.org/10.1016/ j.jrp.2008.12.014

Gosling, S. D., Rentfrow, P. J., \& Swann, W. B. (2003). A very brief measure of the Big Five personality domains. Journal of Research in Personality, 37, 504-528. http://dx.doi.org/10.1016/S0092-6566(03)00046-1

Grant, S., Langan-Fox, J., \& Anglim, J. (2009). The Big Five traits as predictors of subjective and psychological well-being. Psychological Reports, 105(1), 205-231. http://dx.doi.org/10.2466/PR0.105.1.205-231

Haslam, N., Whelan, J., \& Bastian, B. (2009). Big five traits mediate associations between values and subjective well-being. Personality and Individual Differences, 46(1), 40-42. http://dx.doi.org/10.1016/j.paid.2008.09.001

Hauck, N., Machado, W. L., Teixeira, M. A. P., \& Bandeira, D. R. (2012). Evidências de validade de marcadores reduzidos para a avaliação da personalidade no modelo dos Cinco Grandes Fatores. Psicologia: Teoria e Pesquisa, 28, 417-423. http://dx.doi.org/10.1590/S0102-37722012000400007

Hauck, N., Teixeira, M. A. P., Machado, W. L., \& Bandeira, D. R. (2012). Marcadores reduzidos para a avaliação da personalidade em adolescentes. Psico-USF, 17(2), 253-261. http://dx.doi.org/10.1590/S1413-82712012000200009

Hayes, N. \& Joseph, S. (2003). Big 5 Correlates of three measures of subjective well-being. Personality and Individual Differences, 34(4), 723-727. http://dx.doi.org/10.1016/S0191-8869(02)00057-0

Horn, J. L. (1965). A rationale and test for estimating the number of factors in factor analysis. Psychometrika, 30 , 179-185. http://dx.doi.org/10.1007/BF02289447

Hutz, C. S., Nunes, C. H. S. S., Silveira, A. D., Serra, J., Anton, M., \& Wieczorek, L. S. (1998). O desenvolvimento de marcadores para a avaliação da personalidade no Modelo dos Cinco Grandes Fatores. Psicologia: Reflexão e Crítica, 11, 395-409. http://dx.doi.org/10.1590/S0102-79721998000200015 
John, O. P., Angleitner, A., \& Ostendorf, F. (1988). The lexical approach to personality: A historical review of trait taxonomic research. European Journal of Personality, 2, 171-203. http://dx.doi.org/10.1002/per.2410020302

John, O. P., Naumann, L. P., \& Soto, C. J. (2010). Paradigm shift to the integrative Big-Five trait taxonomy: History, measurement, and conceptual issues. In O. P. John, R. W. Robins, \& L. A. Pervin (Eds.), Handbook of personality: Theory and research (3rd ed.) (pp. 114-158). New York: Guilford Press.

Knapp, T. R. (1978). Canonical correlation analysis: A general parametric significance-testing system. Psychological Bulletin, 85(2), 410-416. http://dx.doi.org/10.1037/0033-2909.85.2.410

Little, T. D., Cunningham, W. A., Shahar, G., Widaman, K. F. (2002). To parcel or not to parcel: Exploring the question, weighing the merits. Structural Equation Modeling, 9(2), 151-73. http://dx.doi.org/10.1207/S15328007SEM0902_1

Machado, W. L., Hauck, N. F., Teixeira, M. A. P., \& Bandeira, D. R. (no prelo). Análise de teoria de resposta ao item de marcadores reduzidos da personalidade. Psico (PUCRS).

McCrae, R. R. (2013). Exploring trait assessment of samples, persons, and cultures. Journal of Personality Assessment, 95(6), 556-570. http://dx.doi.org/10.1080/00223891.2013.821075

McCrae, R. R., Terracciano, A., \& 78 Members of the Personality Profiles of Cultures Project. (2005). Universal features of personality traits from the observer's perspective: Data from 50 cultures. Journal of Personality and Social Psychology, 88(3), 547-561. http://dx.doi.org/10.1037/0022-3514.88.3.547

Natividade, J. C., Barros, M. C., \& Hutz, C. S. (2012). Influência da flexão de gênero dos adjetivos em instrumentos psicológicos em português. Avaliação Psicológica, 11(2), 259-264.

Norman, W. T. (1963). Toward an adequate taxonomy of personality attributes: Replicated factor structure in peer nomination personality ratings. Journal of Abnormal and Social Psychology, 66, 574-583. http://dx.doi.org/10.1037/h0040291

Nunes, C. H. S. S. \& Hutz, C. S. (2005). O modelo dos cinco grandes fatores de personalidade. In R. Primi (Ed.), Temas em Avaliação Psicológica (pp. 87-106). São Paulo: Casa do Psicólogo.

Nunes, C. H. S., Hutz, C. S., \& Giacomoni, C. H. (2009). Associação entre bem estar subjetivo e personalidade no modelo dos cinco grandes fatores. Avaliação psicológica, 8(1), 99-108.

Nunes, C. H. S. S., Hutz, C. S., \& Nunes, M. F. O. (2010). Bateria Fatorial de Personalidade. São Paulo: Casa do Psicólogo.

Nunnally, J. C. (1978). Psychometric theory (2nd ed.). New York: McGraw Hill.

Pinho, C. C. M. \& Guzzo, R. S. L. (2003). Taxonomia de adjetivos descritores de personalidade. Avaliação Psicológica, 2(2), 81-97.

R Development Core Team (2012). R: a language and environment for statistical computing. Vienna: R Foundation for Statistical Computing.

Rammstedt, B. \& John, O. P. (2007). Measuring personality in one minute or less: A 10-item short version of the Big Five Inventory in English and German. Journal of Research in Personality, 41, 203-212. http://dx.doi.org/10.1016/ j.jrp.2006.02.001

Rossel, Y. (2012). lavaan: an R package for structural equation modeling. Journal of Statistical Software, 48(2), 1-36.

Satorra, A. \& Bentler, P. M. (1994). Corrections to test statistics and standard errors in covariance structure analysis. In A. von Eye \& C. C. Clogg (Eds.), Latent variables analysis: Applications for developmental research (pp. 399-419). Thousand Oaks, CA: Sage Publications, Inc.

Saucier, G. \& Goldberg, L. R. (1996). The language or personality: Lexical perspective on the Five-Factor model. In J. S. Wiggins (Ed.), The five-factor model of personality: Theoretical perspectives (pp. 21-50). New York: Guilford Press.

Saucier, G. \& Goldberg, L. R. (2001). Lexical studies of indigenous personality factors: Premises, products, and prospects. Journal of Personality, 69, 847-879. http://dx.doi.org/10.1111/1467-6494.696167

Saucier, G., Georgiades, S., Tsaousis, I., \& Goldberg, L. R. (2005). The factor structure of Greek personality adjectives. Journal of Personality and Social Psychology, 88, 856-875. http://dx.doi.org/10.1037/0022-3514.88.5.856

Schmitt, D. P., Realo, A., Voracek, M., \& Allik, J. (2008). Why can't a man be more like a woman? Sex differences in Big Five personality traits across 55 cultures. Journal of Personality and Social Psychology, 94, 168-182. http://dx.doi. org/10.1037/0022-3514.94.1.168

Vianelloa, M., Schnabel, K., Sriram, N., \& Nosek, B. (2013).Gender differences in implicit and explicit personality traits. Personality and Individual Differences, 55(8), 994-999. http://dx.doi.org/10.1016/j.paid.2013.08.008

Woyciekoski, C., Natividade, J. C., \& Hutz, C. S. (no prelo). As contribuições da personalidade e dos eventos de vida para o bem-estar subjetivo. Psicologia: Teoria e Pesquisa (UnB).

Nota:

Um dos autores recebeu bolsa de doutorado da CAPES enquanto realizava pesquisa.

Autores:

JeAn CARLOS NATIVIDADE - Doutor, Universidade Federal do Rio Grande do Sul.

Claudio SimOn HutZ - Doutor, Universidade Federal do Rio Grande do Sul.

Endereço para correspondência:

Jean Carlos Natividade

PUC-Rio - Depto. de Psicologia

Rua Marques de São Vicente, 225

22451-900 Rio de Janeiro, RJ, Brasil

E-mail: jeannatividade@gmail.com

Recebido em: 07.04.14

Aceito em: 03.10.14 